\title{
ARTICLE
}

\section{A microprocessor based on a two-dimensional semiconductor}

\author{
Stefan Wachter ${ }^{1, \star}$, Dmitry K. Polyushkin ${ }^{1, \star}$, Ole Bethge ${ }^{2} \&$ Thomas Mueller $^{1}$
}

The advent of microcomputers in the 1970s has dramatically changed our society. Since then, microprocessors have been made almost exclusively from silicon, but the ever-increasing demand for higher integration density and speed, lower power consumption and better integrability with everyday goods has prompted the search for alternatives. Germanium and III-V compound semiconductors are being considered promising candidates for future high-performance processor generations and chips based on thin-film plastic technology or carbon nanotubes could allow for embedding electronic intelligence into arbitrary objects for the Internet-of-Things. Here, we present a 1-bit implementation of a microprocessor using a two-dimensional semiconductor-molybdenum disulfide. The device can execute user-defined programs stored in an external memory, perform logical operations and communicate with its periphery. Our 1-bit design is readily scalable to multi-bit data. The device consists of 115 transistors and constitutes the most complex circuitry so far made from a two-dimensional material.

\footnotetext{
${ }^{1}$ Institute of Photonics, Vienna University of Technology, Gußhausstraße 27-29, 1040 Vienna, Austria. ${ }^{2}$ Institute of Solid State Electronics, Vienna University of Technology, Floragasse 7, 1040 Vienna, Austria. ${ }^{*}$ These authors contributed equally to this work. Correspondence and requests for materials should be addressed to T.M. (email: thomas.mueller@tuwien.ac.at).
} 
$\mathrm{T}$ wo-dimensional (2D) materials, such as semiconducting transition metal dichalcogenides $(\mathrm{TMDs})^{1,2}$, black phosphorus $^{3}$, silicene ${ }^{4}$ and others, are considered promising candidates for future generations of electronic circuits. It is currently not foreseen that silicon will be replaced for mainstream digital electronics in the mid-term future; however, similar to organic semiconductors ${ }^{5}$ or carbon nanotubes ${ }^{6}, 2 \mathrm{D}$ materials offer a number of interesting properties that could lead to novel applications. Their ultrathin channel thickness provides improved electrostatic gate control and reduced short-channel effects ${ }^{7,8}$, which ultimately results in better geometric scaling behaviour ${ }^{9,10}$ and less power consumption. 2D semiconductors are also one of the leading candidates to enable tunnel field-effect transistors $(\mathrm{FETs})^{11,12}$, working with sub-threshold swing below $60 \mathrm{mV}$ per decade and thus low supply voltage. Together, with their high mechanical flexibility and stability, optical transparency, excellent optoelectronic properties ${ }^{13}$ and compatibility with standard semiconductor technology processing this could lead to energy efficient and flexible electronics ${ }^{14-16}$.

The field of TMD-based electronics has progressed enormously during the past few years. Soon after the first realizations of bulk $^{17,18}$ and monolayer ${ }^{2}$ FETs, basic electronic circuits were demonstrated ${ }^{19,20}$. Both n-type (NMOS) $)^{19-21}$ and complementary $(\mathrm{CMOS})^{22,23}$ metal-oxide-semiconductor technologies have been developed and a good understanding of the FET device physics has been gained ${ }^{24-26}$. The work on devices has been paralleled by the development of growth techniques ${ }^{27-30}$ for the large-scale fabrication of TMD films with good uniformity over the size of a wafer ${ }^{30}$ and the development of technologies for transferring 2D materials onto bendable ${ }^{14-16}$ substrates. Nevertheless, due to the plethora of challenges being faced in large-scale integration, previous work has so far been restricted to applications consisting of only a few transistors and with limited functionality. These challenges range from the necessity to match voltage levels and achieve high noise margins in cascaded logic stages to stringent requirements on device uniformity over millimetre size dimensions.

Here, we demonstrate the feasibility of using a $2 \mathrm{D}$ semiconductor to realize a complex digital circuit-a microprocessor.

\section{Results}

Microprocessor architecture. Figure 1a depicts the architectural block diagram of our microprocessor. For demonstration purposes, we minimized transistor count and thus realized a device that operates on single-bit data only. We stress that this is not a fundamental limitation and the device is readily scalable to $N$-bit data, broadly speaking by connecting $N$ of our devices in parallel. Although we reduced the architecture of our device to the essentials, it comprises all basic building blocks that are common to most microprocessors. In particular, these are: an arithmetic logic unit (ALU), that forms the heart of the processor and is, in general, capable of performing basic arithmetic and logical operations; for simplicity, we have implemented here only logical conjunction and disjunction operations. An accumulator (AC), which holds one of the operands to be supplied to the ALU. An instruction register (IR), that stores the content of the program memory currently being executed, where the most significant two bits contain the instruction itself and the third bit contains the data (Although we retrieve the data directly from the program memory, our device can also process data stored in a separate data memory (Harvard architecture). In this case, the IR is supplied with an address that points to the data memory content, which is then placed on the bus.). A control unit (CU), that receives as input the instruction code from the IR and orchestrates all resources by enabling components to access the internal bus via the control signals $\mathrm{EA}$ and $\mathrm{EO} ; \mathrm{A} / \mathrm{O}$ conveys to the ALU the operation selection code (conjunction, $\mathrm{A} / \mathrm{O}=0$; disjunction, $\mathrm{A} / \mathrm{O}=1)$. A program counter $(\mathrm{PC})$, which supplies the memory with the address of the active instruction. And, finally, an output register (OR), that allows the processor to transfer the results of a calculation to the output port. The memory is, as usual, implemented off-chip.

Figure $1 \mathrm{~b}$ depicts the timing diagram of the device, using three clock (CLK) signals. The execution of each instruction occurs in two sequences - a FETCH sequence followed by an execute (EXE) sequence. The FETCH sequence consists of two phases: in a first phase, the content of the external memory (at the address stored in the PC) is loaded into the IR; the PC is then incremented in a second step. During the EXE sequence, which is implemented here in a single phase, the microprocessor decodes and executes the command stored in the IR. This cycle is repeated continuously. Each phase is triggered by a CLK signal (CLK1, phase 1; CLK2, phase 2; CLK3, phase 3). In order to be flexible in terms of clock rate and timing, we generated the CLK signals externally; an on-chip implementation is straightforward. Figure 1c summarizes the instruction set that we have implemented. The instructions are encoded with two bits; some of them are followed by one bit of data. The no-operation (NOP) instruction has no effect other than to increase the PC. LDA allows the transfer of data from the memory into the AC. AND and OR perform logical conjunction and disjunction operations, respectively.

It is instructive to consider a simple example. The program fragment

$\begin{array}{ccc}\text { Address } & \text { Mnemonic } & \text { OpCode } \\ 0 & \text { LDA 0 } & 010 \\ 1 & \text { AND 1 } & 101\end{array}$

transfers in a first step, triggered by CLK1, the bit sequence 010 from the memory into the IR. CLK2 then increases the $\mathrm{PC}$ and the next instruction becomes available, but is not loaded into the IR yet. Triggered by CLK3, the CU then signals the AC $(E A=1)$ to receive the data $(0)$ from the IR via the internal bus. With the next CLK1 signal, the content of the IR is updated $(\mathrm{IR}=101)$, and the $\mathrm{CU}$ enables the ALU to perform a logical conjunction operation $(\mathrm{A} / \mathrm{O}=0)$ between the data on the bus (1) and that stored in the AC during the previous instruction. Triggered by CLK3, the result of this operation (0) is finally written into the $\mathrm{OR}(\mathrm{EO}=1)$.

Device implementation. We now come to the actual device implementation using a 2D semiconductor. Our microprocessor was fabricated in gate-first technology on a silicon wafer with 280-nm-thick silicon dioxide. The substrate fulfills no other function than acting as a carrier medium and could thus be replaced by glass ${ }^{31}$ or any other material, including flexible substrates $^{14-16}$. We fabricated 18 devices per wafer, with FET channels made from chemical vapour deposition (CVD) grown large-area bilayer $\mathrm{MoS}_{2}$ films. Two Ti/Au metal layers were used to interconnect the transistors and $\mathrm{Al}_{2} \mathrm{O}_{3}$ was used as gate oxide. A detailed description of the device fabrication steps can be found in Methods. Subunits, such as for example, the ALU or the IR, were provided with metal pads for individual testing in a wafer probe station. All subunits were eventually bonded together and the sample was placed back into the probe chamber, where it remained in vacuum for final testing of the complete circuit.

Figure $2 \mathrm{a}$ (bottom) shows a schematic drawing of a so-obtained $\mathrm{MoS}_{2}$ FET. The devices exhibit a field-effect mobility of $\sim 3 \mathrm{~cm}^{2} \mathrm{~V}^{-1} \mathrm{~s}^{-1}$, a threshold voltage $V_{\mathrm{T}}$ of $\sim 0.65 \mathrm{~V}$ 
a

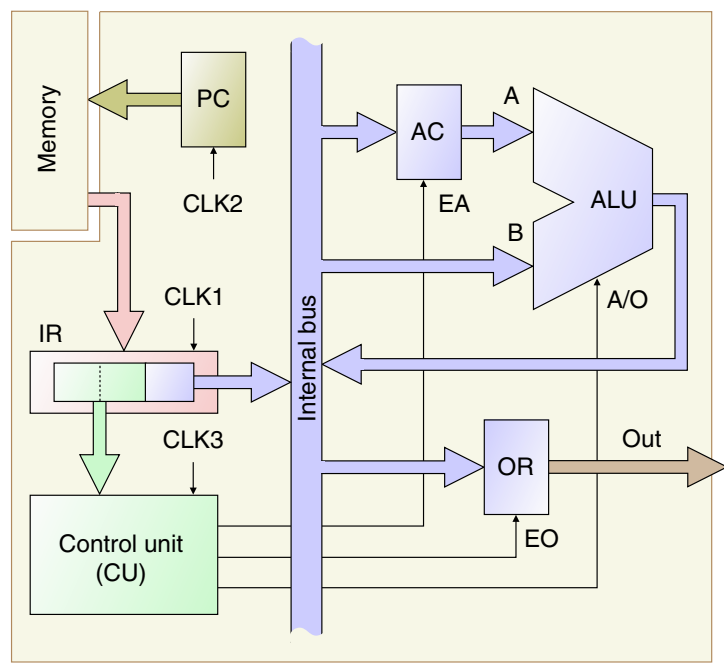

b

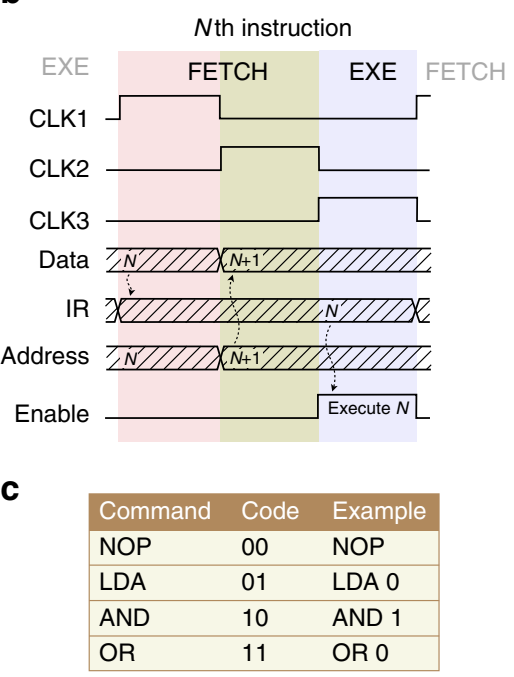

Figure 1 | Microprocessor architecture. (a) Block diagram, showing the arithmetic logic unit (ALU) with inputs $A$ and $B$, accumulator ( $A C$ ), control unit $(C U)$, instruction register (IR), output register (OR) and program counter (PC). Enable signals (EA and EO) and operation selection code (A/O) are supplied by the $\mathrm{CU}$ to the respective subunits. CLK signal generation and memory are implemented off-chip. (b) Timing diagram for the Nth instruction cycle. During the FETCH sequence the content of the memory is loaded into the IR and the address, stored in the PC, is increased. During the EXE sequence the command, stored in the IR, is executed. (c) Instruction set of the microprocessor. NOP is the no-operation instruction; LDA transfers data from the memory into the AC; AND and OR perform logical operations.

a

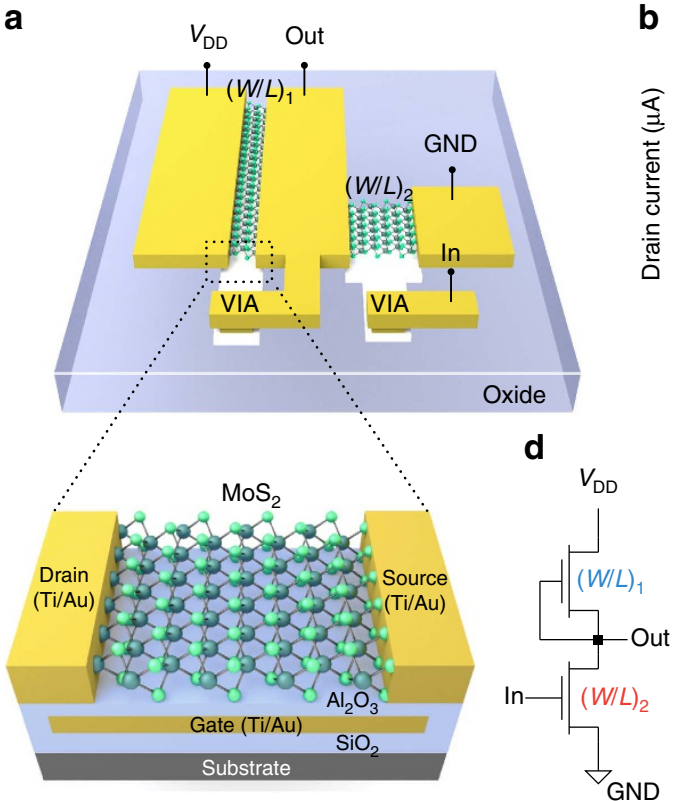

b
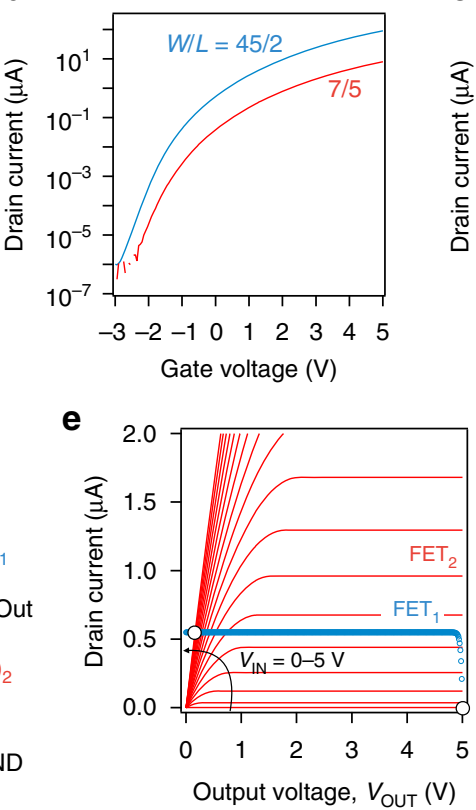

C

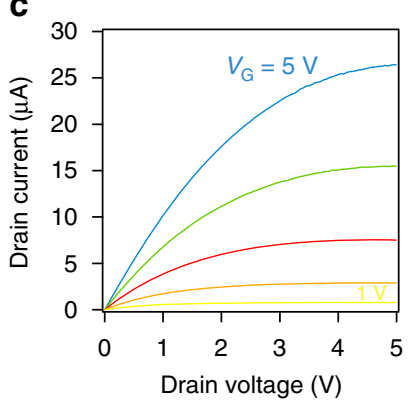

f

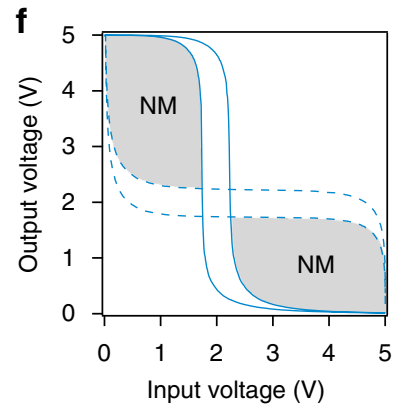

Figure 2 | Characterization of $\mathbf{M o S}_{\mathbf{2}}$ transistors and inverter. (a) Schematic drawing of an inverter circuit (top) and an individual MoS 2 transistor (bottom) in gate-first technology (see Supplementary Fig. 5 for corresponding micrograph). (b) Transfer characteristics of load (W/L=45/2) and pull-down $(W / L=7 / 5)$ transistors. (c) Output characteristic for gate voltages between 1 and $5 \mathrm{~V}$ (in $1 \mathrm{~V}$ steps). (d) NMOS inverter circuit schematic. (e) Graphical construction to determine the output voltage $V_{\text {OUT }}$ of an inverter for a given input voltage $V_{\text {IN }}$. The blue symbols show the load curve and the red lines are the output characteristics of the pull-down transistor (in $0.25 \mathrm{~V}$ steps). The intersection point of both curves determines $V_{\text {OUT. }}$ (f) The solid line shows the measured voltage transfer characteristic of an inverter. By mirroring this curve (dashed line) a butterfly plot is obtained, from which NM can be extracted by nesting the largest possible square in the grey shaded area.

(Supplementary Fig. 3), an on/off ratio of $\sim 10^{8}$, and uniform behaviour over a $\sim 50 \mathrm{~mm}^{2}$ area over the wafer (Supplementary Fig. 4). The circuit is based on the NMOS logic family, where both pull-up (load) and pull-down networks were realized using n-type enhancement-mode FETs. The implementation of an inverter (see circuit schematic in Fig. 2d) using this logic family is shown in Fig. 2a (top). A careful design of the $W / L$ ratios, where $W$ and $L$ denote the width and length of the FET channels, is crucial, as it determines the switching threshold voltage $V_{\mathrm{M}}$ and thus the ability to cascade logic stages. For simple analytic 


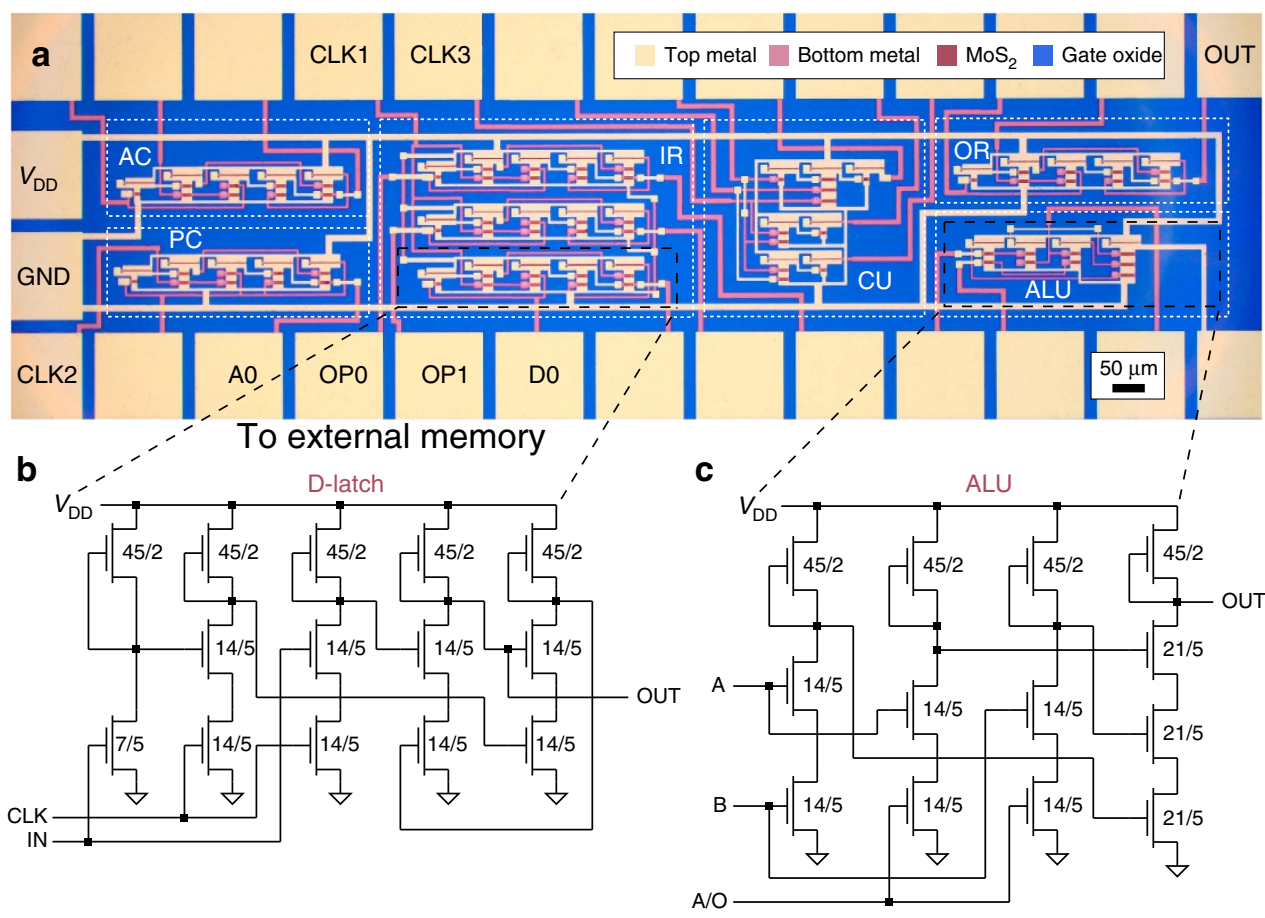

Figure 3 | Device implementation using a 2D semiconductor. (a) Microscope image of the microprocessor. The two metal layers appear in different colour and are connected with via-holes. All subunits were provided with metal pads for individual testing. Labelled pads were used to connect the device to the periphery (memory, CLK signal generation, power supply, output), the others were wire bonded together to realize the internal connections. Scale bar, $50 \mu \mathrm{m}$. Circuit schematics of (b) D-Latch and (c) ALU, with $W / L$ ratio in units of $\mu \mathrm{m} / \mu \mathrm{m}$ for each transistor. IN, input; OUT, output. The complete microprocessor schematic is presented in Supplementary Fig. 1.

modelling, we performed calculations based on long-channel FET theory ${ }^{32}$. The pull-down FET is described by $I_{\mathrm{D} 2}=K_{2}\left[\left(V_{\mathrm{IN}}-V_{\mathrm{T}}\right) V_{\mathrm{OUT}}-V_{\mathrm{OUT}}^{2} / 2\right]$ in the triode regime and $I_{\mathrm{D} 2}=K_{2}\left(V_{\mathrm{IN}}-V_{\mathrm{T}}\right)^{2} / 2$ in the saturation regime (red curves in Fig. 2e). The load FET is operated in the sub-threshold regime $\left(V_{\mathrm{G} 1}=0<V_{\mathrm{T}}\right)$, and thus acts as a current source over a large drain voltage range, $I_{\mathrm{D} 1}=K_{1}\left[1-\exp \left(-\beta V_{\mathrm{D} 1}\right)\right]$ with $\beta$ being the reciprocal of the thermal potential. From the circuit schematic Fig. $2 \mathrm{~d}$, it is apparent that $V_{\mathrm{D} 1}=V_{\mathrm{DD}}-V_{\mathrm{OUT}}$, and thus $I_{\mathrm{D} 1}=K_{1}\left[1-\exp \left(\beta V_{\mathrm{OUT}}-\beta V_{\mathrm{DD}}\right)\right]$ (blue symbols in Fig. 2e). The parameters $K_{1}$ and $K_{2}$ are taken from the experiment (Fig. 2b). By equating both currents, $I_{\mathrm{D} 1}=I_{\mathrm{D} 2}$, we obtain a relation between $V_{\text {OUT }}$ and $V_{\mathrm{IN}}$, from which the switching threshold $V_{\mathrm{M}}$ can be determined (Supplementary Fig. 6). If both transistors are implemented with same $W / L$ ratio, $V_{\mathrm{M}}$ drops below $1 \mathrm{~V}$ (Supplementary Fig. 6b), resulting in low noise margin, especially in the presence of additional hysteresis. Asymmetric transistor design, on the other hand, allows shifting $V_{\mathrm{M}}$ towards $V_{\mathrm{DD}} / 2$ (Supplementary Fig. 6a), resulting in improved switching behaviour. $W / L$ ratios of the pull-up and pull-down transistors were hence made $45 / 2(\mu \mathrm{m} / \mu \mathrm{m})$ and $7 / 5$, respectively.

Logic NAND gates with $M$ inputs were implemented by connecting $M$ pull-down transistors with $W / L=(M \times 7) / 5$ in series. The processor was realized by using a combination of these elements. The minimum feature size of $2 \mu \mathrm{m}$ was chosen rather large for two reasons. It makes the design immune to sample inhomogeneities (for example, small holes, cracks and contaminations in the $\mathrm{MoS}_{2}$ film) and also allows for fast visual inspection of the lithographic structures with an optical microscope. Because of the immunity of $2 \mathrm{D}$ transistors to short-channel effects ${ }^{7-10}$, we expect comparable performance when the devices are scaled to sub-micrometre dimensions, provided that low contact resistance can be achieved.
Figure $2 \mathrm{~b}$ shows the transfer characteristics of load and pulldown transistors, where the $\sim 14$ times higher current through the former demonstrates reliable controllability of the device characteristics by geometrical scaling. The output characteristic, depicted in Fig. 2c, shows clear current saturation due to channel pinch-off at the drain. The voltage transfer characteristic of our inverters exhibit excellent performance for a wide supply voltage range between $V_{\mathrm{DD}}=2$ and $7 \mathrm{~V}$, with input and output logic levels being perfectly matched. Figure $2 \mathrm{f}$ (solid line) shows the results for $V_{\mathrm{DD}}=5 \mathrm{~V}$, for which the voltage gain $A_{\mathrm{V}}=-\mathrm{d} V_{\text {OUT }} / \mathrm{d} V_{\text {IN }}$ reaches values of $A_{\mathrm{V}} \approx 60$. Although the voltage transfer curve shows some hysteresis (that mostly stems from trap charges in the gate oxide) the noise margin of the inverter (see shaded area in Fig. $2 \mathrm{f}), \mathrm{NM} \approx 0.59 \times\left(V_{\mathrm{DD}} / 2\right)$, is sufficiently large for integration into multi-stage logic circuits. The NAND gates showed comparable performance. We estimate a static power consumption of $P_{\mathrm{s}}=V_{\mathrm{DD}}\left(I_{\mathrm{D}, \mathrm{L}}+I_{\mathrm{D}, \mathrm{H}}\right) / 2 \approx 1.4 \mu \mathrm{W}$ per logic gate, where $I_{\mathrm{D}, \mathrm{L}}$ and $I_{\mathrm{D}, \mathrm{H}}$ denote the currents at $V_{\mathrm{IN}}=0$ and $5 \mathrm{~V}$ (Fig. 2e), respectively. The total power consumption of the circuit, consisting of 41 stages, is thus $\sim 60 \mu \mathrm{W}$.

A microscope image of the microprocessor is shown in Fig. 3a. The device is composed of $115 \mathrm{MoS}_{2}$ transistors and measureswithout bonding pads $-0.6 \mathrm{~mm}^{2}$ in size. Circuit schematics for a D-Latch and the ALU are shown in Fig. 3b,c, respectively. The complete schematic is presented in Supplementary Fig. 1. A D-Latch is a bi-stable circuit that can be used as 1-bit data storage element, triggered by a CLK signal. It forms the basic building block of all our data registers (IR, AC and OR) and the PC. The ALU is a combinational logic circuit, entirely based on NANDs, that performs bitwise logic operations on 1-bit data. The additional input A/O signals the ALU which operation to perform. Measurements of the ALU output for different input logic states are presented in Supplementary Fig. 8. 
a

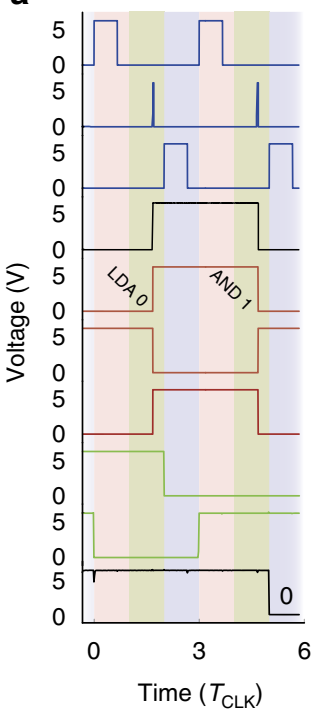

b

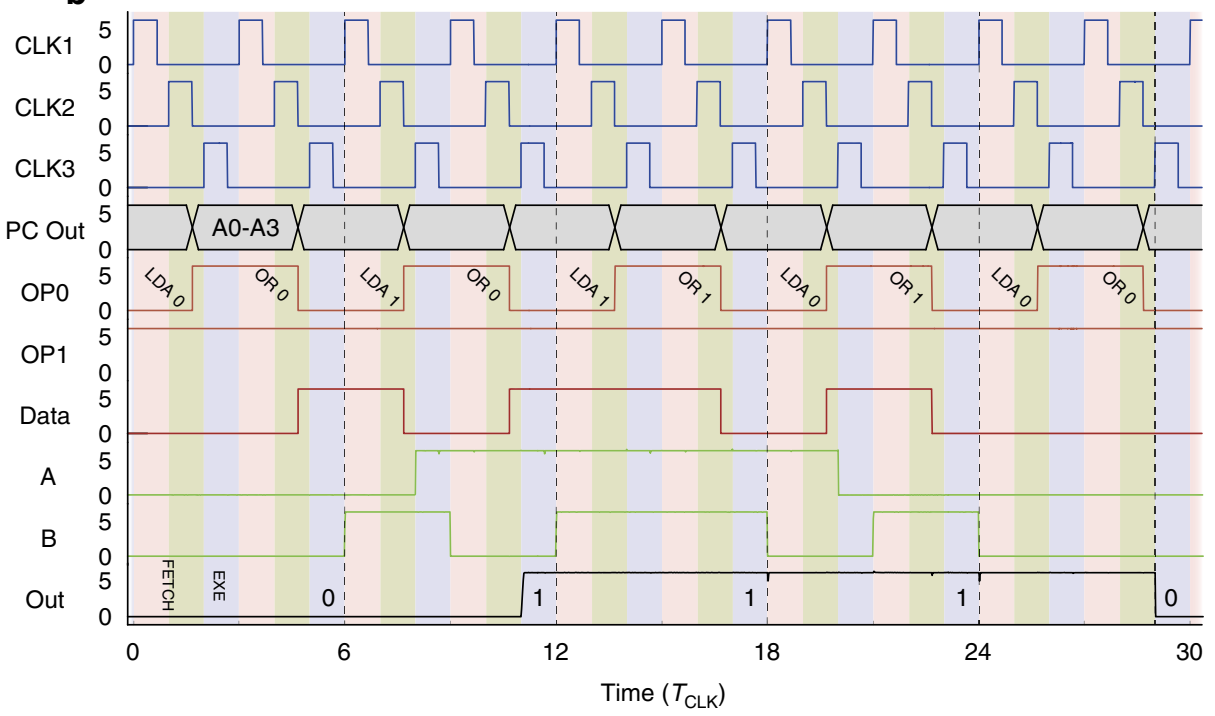

Figure 4 | Device operation. (a) Waveforms measured on the chip when running the sample program, explained above. The CLK1, CLK2 and CKL3 signals were generated externally. Each instruction requires three CLK cycles, where $1 / T_{\text {CLK }}$ is the CLK frequency. CLK2 pulses were made sufficiently short to trigger the level-sensitive input of the PC; this could be avoided by using a more complex master-slave design (Supplementary Fig. 9). AO is the address supplied by the PC. OPO, OP1 and DO denote the signals from the memory, where the former two are the instruction and the latter is the data. A and B are the input signals to the ALU, and OUT is the output of the device. $T_{C L K}=500 \mathrm{~ms}$. (b) Results for a series of other calculations. In order to run the longer program, a 4-bit PC was implemented externally. The meaning of the curves is the same as in a, apart from AO which schematically depicts here the 4-bit address signal (A0-A3). Again, the device is able to deliver the expected logic values, shown as numbers at the bottom.

We first verified the functionality of the microprocessor by running the example program from above and measuring waveforms at different locations on the chip (see Methods for measurement details). As shown in Fig. 4a, the device is indeed able to deliver the correct result, with excellent signal integrity and with rail-to-rail performance, proving the ability to cascade logic stages based on 2D semiconductors. To further demonstrate the operability of the device, we present in Fig. $4 \mathrm{~b}$ the results from a series of logical disjunction operations. The match of measured and expected outputs shows again correct operation. As shown in Supplementary Fig. 10, the device proved to be functional at CLK frequencies of $50 \mathrm{~Hz}$. This is by no means a limitation of the TMD material itself, but is caused by the limitations of our measurement setup. Ultimately, the speed is limited by the current-driving capability of the pull-up transistor, which is operated in the sub-threshold regime $\left(V_{\mathrm{GS}}=0<V_{\mathrm{T}}\right)$ and acts as current source with $I_{\mathrm{D}} \approx 0.55 \mu \mathrm{A}$. For a typical (external) capacitive load of $C_{\mathrm{L}} \approx 1-10 \mathrm{pF}$, we estimate a maximum operation frequency of $f_{\mathrm{MAX}} \approx I_{\mathrm{D}} /\left(2 \pi V_{\mathrm{DD}} C_{\mathrm{L}}\right) \approx 2-20 \mathrm{kHz}$ (Supplementary Fig. 11). To increase $f_{\mathrm{MAX}}, I_{\mathrm{D}}$ could be increased by employing depletion-mode load FETs ${ }^{20}$, controlled chemical doping, improving the carrier mobility of the $2 \mathrm{D}$ semiconductor or just by reducing the transistor channel lengths.

\section{Discussion}

In summary, we have reported a first step towards the development of microprocessors based on $2 \mathrm{D}$ semiconductors. The major challenge that we faced during device fabrication is yield. Although the yield for subunits was high (for example, $\sim 80 \%$ of ALUs were fully functional), the sheer complexity of the full system, together with the non-fault tolerant design, resulted in an overall yield of only a few per cent of fully functional devices. Imperfections of the $\mathrm{MoS}_{2}$ film, mainly caused by the transfer from the growth to the target substrate, were identified as main source for device failure. However, as no metal catalyst is required for the synthesis of TMD films ${ }^{27-30}$, direct growth on the target substrate is a promising route to improve yield. Besides that, we do not see any roadblocks that could prevent the scaling of our 1-bit design to multi-bit data. Our work demonstrates that integrated circuits consisting of $2 \mathrm{D}$ materials are a promising emerging technology.

\section{Methods}

Device fabrication. Fabrication (Supplementary Fig. 7) started with patterning of the bottom metal (gate) layer by electron beam lithography (EBL) and evaporation of Ti/Au (5/25 nm). A 22-nm-thick $\mathrm{Al}_{2} \mathrm{O}_{3}$ gate oxide was then deposited using atomic layer deposition, followed by a second lithography step and wet chemical etching in potassium hydroxide to define the via-holes that connect the bottom and top metal layers where necessary. Following the procedure described in ref. 29, a large-area $\mathrm{MoS}_{2}$ film was grown by CVD on sapphire and then transferred onto the target wafer. The film is continuous over an area of $\sim 50 \mathrm{~mm}^{2}$ with bilayer thickness and small multi-layer $\mathrm{MoS}_{2}$ islands and contaminations. The $\mathrm{MoS}_{2}$ film was characterized by atomic force microscopy and Raman spectroscopy (Supplementary Fig. 2). In a third EBL step, rectangular $\mathrm{MoS}_{2}$ channels were patterned and subsequently etched using Ar/SF6 plasma. Before lift-off, mild treatment of the sample in oxygen plasma was performed to remove the crust from the surface of the polymer mask. The top metal (drain/source contact) layer was then formed by another EBL process and subsequent $\mathrm{Ti} / \mathrm{Au}(5 / 35 \mathrm{~nm})$ deposition. The sample was finally annealed in vacuum at $400 \mathrm{~K}$ for several hours to remove adsorbants from the surface and reduce device hysteresis.

Electrical testing. For testing, we generated the CLK signals externally, using a digital I/O card (National Instruments PCI-6229) in a computer. The same card was used for emulating the external memory. The device was supplied with $V_{\mathrm{DD}}=5 \mathrm{~V}$, and waveforms were recorded with a Semiconductor Parameter Analyzer (Agilent $4155 \mathrm{C}$ ), connected to the probe tips of a wafer probe station (Lakeshore TTPX).

Data availability. The data that support the findings of this study are available from the corresponding author on request.

\section{References}

1. Mak, K. F., Lee, C., Hone, J., Shan, J. \& Heinz, T. F. Atomically thin $\mathrm{MoS}_{2}$ : a new direct-gap semiconductor. Phys. Rev. Lett. 105, 136805 (2010).

2. Radisavljevic, B., Radenovic, A., Brivio, J., Giacometti, V. \& Kis, A. Single-layer $\mathrm{MoS}_{2}$ transistors. Nat. Nanotechnol. 6, 147-150 (2011). 
3. Li, L. et al. Black phosphorus field-effect transistors. Nat. Nanotechnol. 9, 372-377 (2014).

4. Tao, L. et al. Silicene field-effect transistors operating at room temperature. Nat. Nanotechnol. 10, 227-231 (2015).

5. Myny, K. et al. An 8-bit, 40-instructions-per-second organic microprocessor on plastic foil. IEEE J. Solid-State Circuits 47, 284-291 (2012).

6. Shulaker, M. M. et al. Carbon nanotube computer. Nature 501, 526-530 (2013).

7. Yan, R. H., Ourmazd, A. \& Lee, K. F. Scaling the Si MOSFET: from bulk to SOI to bulk. IEEE Trans. Electron Dev. 39, 1704-1710 (1992).

8. Fiori, G. et al. Electronics based on two-dimensional materials. Nat. Nanotechnol. 9, 768-779 (2014).

9. Liu, H., Neal, A. T. \& Ye, P. D. Channel length scaling of $\mathrm{MoS}_{2}$ MOSFETs. ACS Nano 6, 8563-8569 (2012).

10. Desai, S. B. et al. $\mathrm{MoS}_{2}$ transistors with 1-nanometer gate lengths. Science 354, 99-102 (2016).

11. Ilatikhameneh, H. et al. Tunnel field-effect transistors in 2-D transition metal dichalcogenide materials. IEEE J. Explor. Solid-State Computat. Devices Circuits 1, 12-18 (2015).

12. Roy, T. et al. Dual-gated $\mathrm{MoS}_{2} / \mathrm{WSe}_{2}$ van der Waals tunnel diodes and transistors. ACS Nano 9, 2071-2079 (2015).

13. Pospischil, A. \& Mueller, T. Optoelectronic devices based on atomically thin transition metal dichalcogenides. Appl. Sci. 6, 78 (2016).

14. $\mathrm{Pu}$, J. et al. Highly flexible $\mathrm{MoS}_{2}$ thin-film transistors with ion gel dielectrics. Nano Lett. 12, 4013-4017 (2012).

15. Lee, G.-H. et al. Flexible and transparent $\mathrm{MoS}_{2}$ field-effect transistors on hexagonal boron nitride-graphene heterostructures. ACS Nano 7, 7931-7936 (2013).

16. Cheng, R. et al. Few-layer molybdenum disulfide transistors and circuits for high-speed flexible electronics. Nat. Commun. 5, 5143 (2014).

17. Podzorov, V., Gershenson, M. E., Kloc, Ch, Zeis, R. \& Bucher, E. High-mobility field-effect transistors based on transition metal dichalcogenides. Appl. Phys. Lett. 84, 3301-3303 (2004).

18. Ayari, A., Cobas, E., Ogundadegbe, O. \& Fuhrer, M. S. Realization and electrical characterization of ultrathin crystals of layered transition- metal dichalcogenides. J. Appl. Phys. 101, 014507 (2007).

19. Radisavljevic, B., Whitwick, M. B. \& Kis, A. Integrated circuits and logic operations based on single-layer $\mathrm{MoS}_{2}$. Nano Lett. 5, 9934-9938 (2011).

20. Wang, H. et al. Integrated circuits based on bilayer $\mathrm{MoS}_{2}$ transistors. Nano Lett. 12, 4674-4680 (2012).

21. Yu, L. et al. Design, modeling and fabrication of CVD grown $\mathrm{MoS}_{2}$ circuits with E-mode FETs for large-area electronics. Nano Lett. 16, 6349-6356 (2016).

22. Tosun, M. et al. High-gain inverters based on WSe $\mathrm{W}_{2}$ complementary field-effect transistors. ACS Nano 8, 4948-4953 (2014).

23. Yu, L. et al. High-performance WSe $\mathrm{W}_{2}$ complementary metal oxide semiconductor technology and integrated circuits. Nano Lett. 15, 4928-4934 (2015).

24. Kim, S. et al. High-mobility and low-power thin-film transistors based on multilayer $\mathrm{MoS}_{2}$ crystals. Nat. Commun. 3, 1011 (2012).

25. Das, S., Chen, H.-Y., Penumatcha, A. V. \& Appenzeller, J. High performance multilayer $\mathrm{MoS}_{2}$ transistors with scandium contacts. Nano Lett. 13, 100-105 (2013).

26. Schwierz, F., Pezoldt, J. \& Granzner, R. Two-dimensional materials and their prospects in transistor electronics. Nanoscale 7, 8261-8283 (2015).
27. Liu, K.-K. et al. Growth of large-area and highly crystalline $\mathrm{MoS}_{2}$ thin layers on insulating substrates. Nano Lett. 12, 1538-1544 (2012).

28. Zhan, Y., Liu, Z., Najmaei, S., Ajayan, P. M. \& Lou, J. Large-area vapor-phase growth and characterization of $\mathrm{MoS}_{2}$ atomic layers on a $\mathrm{SiO}_{2}$ substrate. Small 8, 966-971 (2012).

29. Dumcenco, D. et al. Large-area epitaxial monolayer $\mathrm{MoS}_{2}$. ACS Nano 9, 4611-4620 (2015).

30. Kang, K. et al. High-mobility three-atom-thick semiconducting films with wafer-scale homogeneity. Nature 520, 656-660 (2015).

31. Kwon, H. et al. Large scale $\mathrm{MoS}_{2}$ nanosheet logic circuits integrated by photolithography on glass. 2D Mater. 3, 044001 (2016).

32. Sze, S. M. Physics of Semiconductor Devices (Wiley-Interscience, 1981).

\section{Acknowledgements}

We thank Simone Schuler, Andreas Pospischil, Marco Furchi, Andreas Kleinl, Fabian Doná, Werner Schrenk, Markus Schinnerl, Peter Kröll and Benedikt Gottsbachner for technical assistance, Alois Lugstein and Emmerich Bertagnolli for providing access to CVD and atomic layer deposition systems, and Dumitru Dumcenco for helpful discussions. We acknowledge financial support by the Austrian Science Fund FWF (START Y 539-N16) and the European Union (Grant agreement No. 696656 Graphene Flagship).

\section{Author contributions}

T.M. conceived the experiment. S.W. and T.M. designed the circuit. D.K.P. grew the $\mathrm{MoS}_{2}$ films. S.W. and D.K.P. fabricated the devices and carried out the measurements. O.B. contributed to the sample fabrication. T.M. prepared the manuscript. All authors discussed the results and commented on the manuscript.

\section{Additional information}

Supplementary Information accompanies this paper at http://www.nature.com/ naturecommunications

Competing interests: The authors declare no competing financial interests.

Reprints and permission information is available online at http://npg.nature.com/ reprintsandpermissions/

How to cite this article: Wachter, S. et al. A microprocessor based on a two-dimensional semiconductor. Nat. Commun. 8, 14948 doi: 10.1038/ncomms14948 (2017).

Publisher's note: Springer Nature remains neutral with regard to jurisdictional claims in published maps and institutional affiliations.

This work is licensed under a Creative Commons Attribution 4.0 International License. The images or other third party material in this article are included in the article's Creative Commons license, unless indicated otherwise in the credit line; if the material is not included under the Creative Commons license, users will need to obtain permission from the license holder to reproduce the material. To view a copy of this license, visit http://creativecommons.org/licenses/by/4.0/

(C) The Author(s) 2017 